\title{
Platelet Rich Fibrin as Wound Coverage of Donor Site in Free Gingival Graft
}

\author{
Dr. Shweta Agrawal, ${ }^{1}$ Dr. Pramod Kumar Koirala, ${ }^{1}$ Dr. Shaili Pradhan, ${ }^{1}$ Dr. Ranjita Shrestha Gorkhali ${ }^{1}$ \\ ${ }^{1}$ Periodontology and Oral Implantology Unit, Department of Dental Surgery, \\ National Academy of Medical Sciences, Bir Hospital, Kathmandu, Nepal.
}

\begin{abstract}
Free gingival graft is one of the technique used for root coverage so as to increase the inadequate keratinized tissue. The donor tissue is most frequently taken from the palate and generally the donor site is not covered or protected. Platelet rich fibrin is one of the good option for the wound coverage of donor site after free gingival graft so as to reduce the pain perception to patient and faster wound healing. The case report includes two cases with gingival recession, treated on the same day with free gingival graft with the use of platelet rich fibrin in the donor site in case 1 and without platelet rich fibrin in case 2 respectively which were compared for pain perception and wound healing postoperatively at 24 hours, 3 days, 5 days, 7 days and 14 days. There was less pain perception and discomfort in the donor site with the use of platelet rich fibrin and enhanced healing compared to donor site without platelet rich fibrin.

Keywords: Donor site; free gingival graft; platelet rich fibrin.
\end{abstract}

\section{INTRODUCTION}

Free gingival graft (FGG) was initially described by Bjorn, in 1963. King and Pennel in 1964 described its clinical applications to correct mucogingival problems. ${ }^{1}$ The term free gingival graft was first suggested by Nabers. ${ }^{1}$ The advantages of using an FGG technique are high predictability and relative ease of technique. The healing process at the donor site proceeds by secondary intention or "granulating in" and takes about two to four weeks depending on the width and thickness of the tissue removed. Although the postoperative response at the donor site is generally uneventful, many patients suffer traumatic and postoperative discomfort with occasional bleeding and delayed healing. ${ }^{1}$ Rossman and Rees had suggested the use of a homeostatic dressing. ${ }^{2}$ Plateletrich fibrin (PRF) was first developed in France by Choukroun et al in 2001. ${ }^{3}$ PRF is an immune and platelet concentrate collecting on a single fibrin membrane all the constituents of a blood sample favorable to healing and immunity. ${ }^{4}$

PRF is a three-dimensional fibrin network that promotes accelerated wound healing, immunity and effective neovascularization, and it supports the three crucial steps

\section{Correspondence:}

Dr. Shweta Agrawal

Periodontology and Oral Implantology Unit, Department of Dental Surgery, National Academy of Medical Sciences, Bir Hospital, Kathmandu, Nepal. email: ashweta.garg@gmail.com

\section{Citation}

Agrawal S, Koirala PK, Pradhan S, Shrestha R, Platelet Rich Fibrin as Wound Coverage of Donor Site in Free Gingival Graft. J Nepal Soc Perio Oral Implantol. 2018;2(1):26-9 of wound healing: 'angiogenesis,' 'immunity' and 'epithelial proliferation. ${ }^{5}$

\section{CASE REPORT 1}

A 25-year-old female with no significant medical history presented inadequate attached gingiva with respect to 34 . Free gingival graft was planned to increase the attached gingival and the patient was prepared for surgery. Local anaesthesia (2\% lignocaine with adrenaline) was given in the recipient site and donor site. Recipient site was prepared with respect to 34, and the measurement for the amount of graft needed was done and leadfoil template was prepared accordingly. Free gingival graft was taken from palatal aspect in respect to 25, 26 and 27, with the use of template. Graft was placed in the recipient site and sutured with 3-0 silk suture (horizontal, circumferential and interdental concavity suture) and periodontal dressing was applied.

The PRF was prepared following the protocol developed by Choukroun et al (2001). Immediately before surgery, intravenous blood alone (without anticoagulant) was collected (by venipuncturing of the antecubital vein) in a sterile 10$\mathrm{ml}$ tube and centrifugation (R-8C, REMI, Mumbai, India) was carried out immediately at $3000 \mathrm{rpm}$ (approximately $400 \mathrm{~g}$ ) for $13 \mathrm{~min}$. Because of differential densities, it result in the separation of three basic fractions. Topmost layer consist of acellular platelet poor plasma, middle layer consist of PRF and the bottom layer had the Red Blood Cells (RBCs). A total of 2-3 $\mathrm{ml}$ of the top layer was pipetted out with the sterile dropper; the middle layer (PRF) was removed and placed in a sterile dappen dish for use. PRF membrane was prepared by squeezing the middle layer between the two glass-slab. 
The PRF membrane was placed on the donor site and sutured with 3-0 silk suture. Hawley's retainer was given.

\section{CASE REPORT 2}

A 27-year-old female with no significant medical history presented with Miller's class II recession with respect to 31 . Free gingival graft was planned for root coverage and the patient was prepared for surgery. Local anaesthesia (2\% lignocaine with adrenaline) was given in both recipient site and donor site. Recipient site was prepared in respect to 31 , and the measurement for the amount of graft needed was done and leadfoil template was prepared accordingly. Free gingival graft was taken from palatal aspect in respect to 25,
26 and 27, with the use of template. Graft was placed in the recipient site and sutured with 3-0 silk suture (horizontal, circumferential and interdental concavity suture) and periodontal dressing was applied. Donor site was secured with periodontal pack supported by Hawley's retainer.

In both cases, patients were advised to rinse with chlorhexidine gluconate mouthrinse (0.2\%) twice daily for a period of 15 days. At 1 week postoperatively, periodontal dressing and sutures were removed. Povidine-iodine solution was used to rinse the surgical site. Both the patients were recalled in same intervals for the evaluation. Patient discomfort and wound healing were recorded after 24 hours, 3 days, 5 days, 7 days and 14 days.
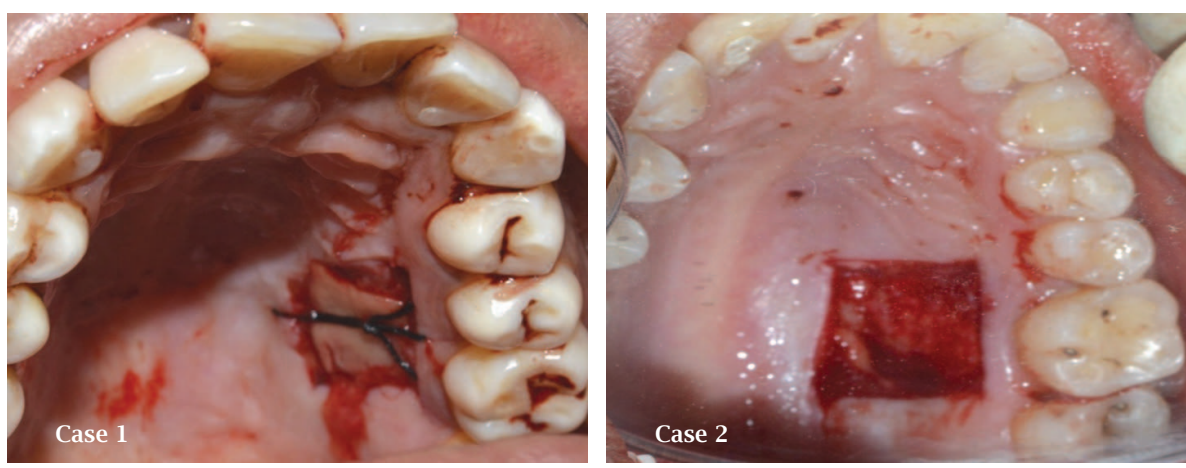

Figure 1: Immediately after surgery.
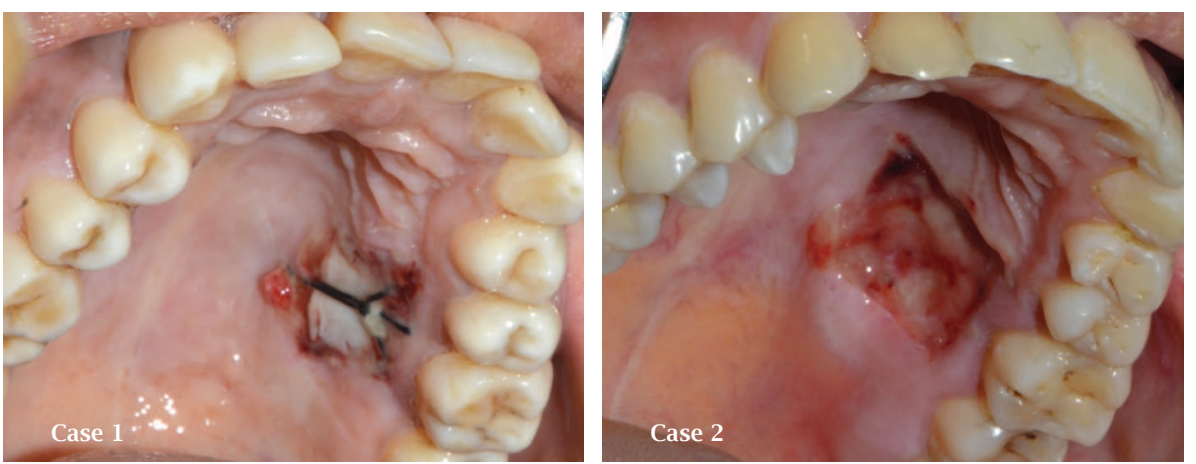

Figure 2: After 24 hours of surgery.
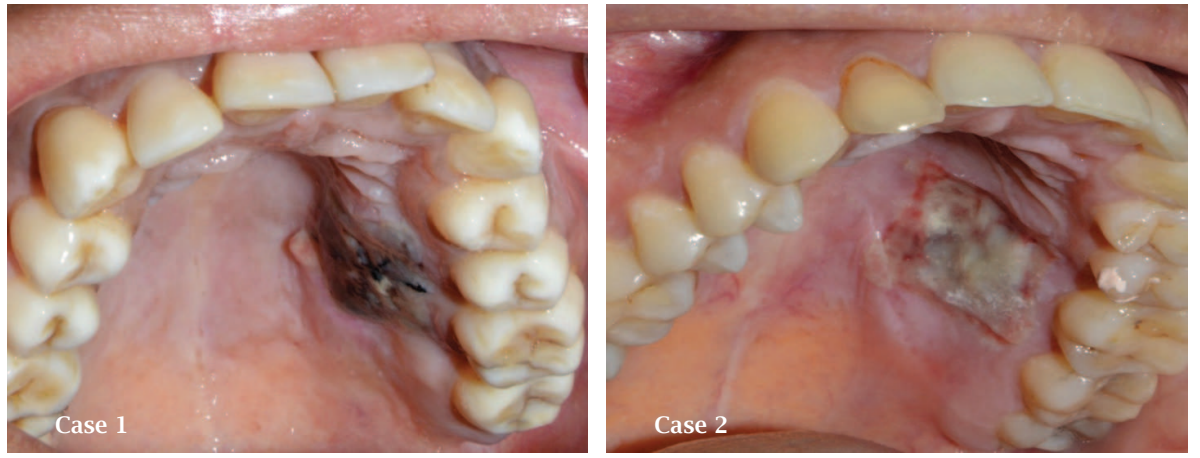

Figure 3: After 3 days of surgery. 

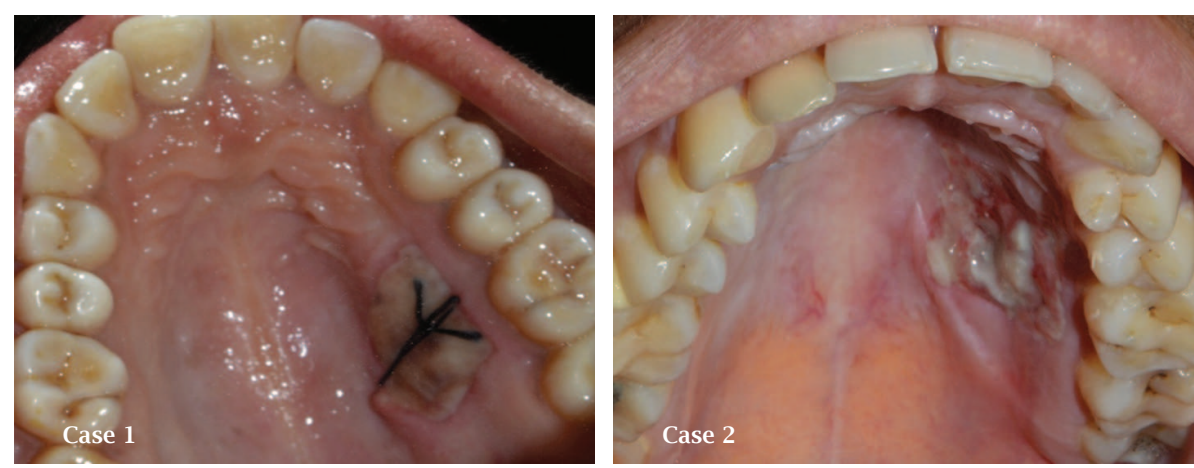

Figure 4: After 5 days of surgery.
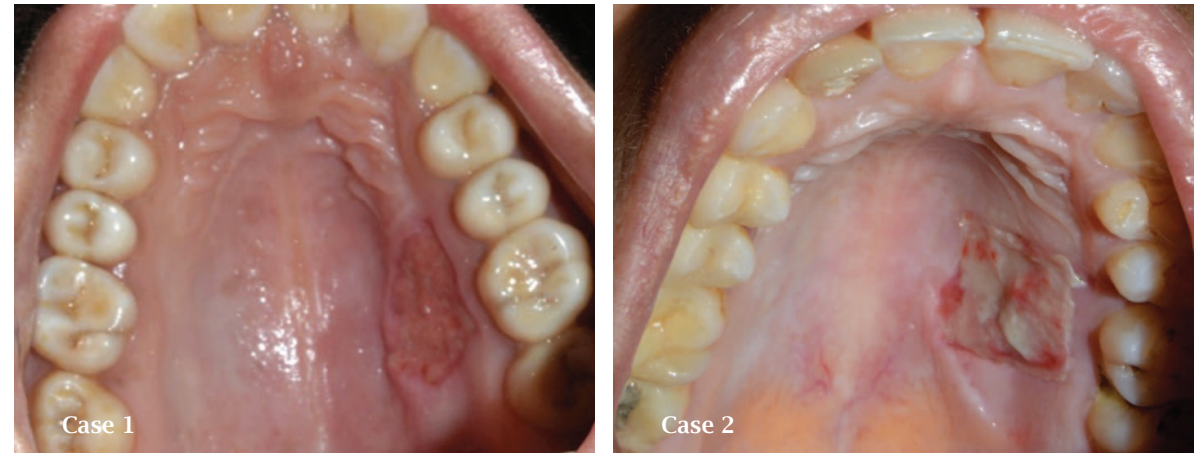

Figure 5: After 7 days of surgery.
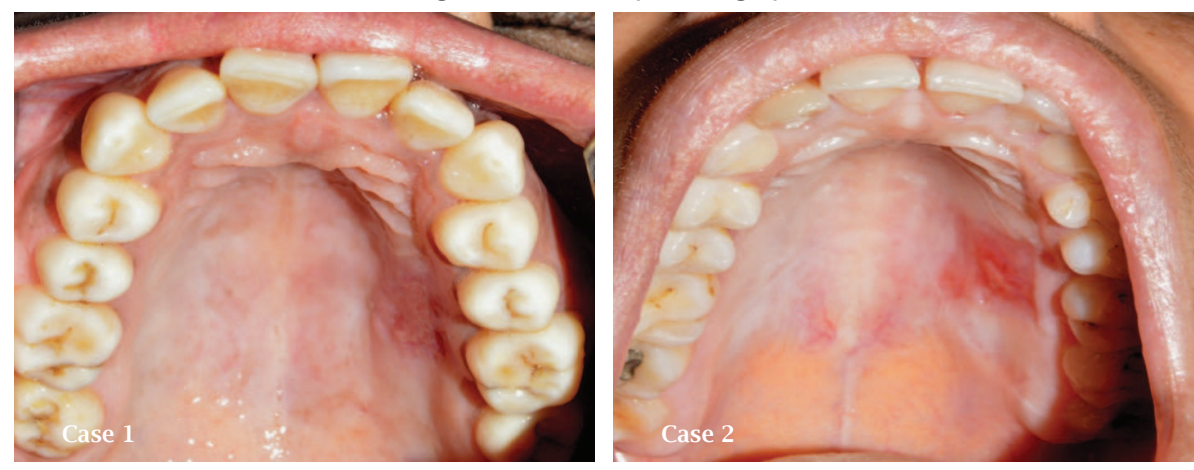

Figure 6: After 14 days of surgery.

\section{RESULT}

Placement of PRF protected the donor site better than leaving the area raw (Figure 1) and Hawley's retainer application caused less discomfort. After 24 hours of postoperative (Figure 2), patient in case 1 had reported less discomfort and pain on keeping and removal of Hawley's retainer than it was with case 2. Similarly, patient with PRF on donor site (case 1) need not take analgesic as compared to that of patient without PRF on the donor site (case 2). After 3 days (Figure 3), the donor site with PRF had better clinical healing as compared to that of patient in case 2. Patient with PRF on donor site had less difficulty while eating compared to the patient without PRF on the donor site. After 5 days, 7 days and 14 days (Figure 4, 5 and 6), the clinical healing of the donor site with PRF was enhanced compared to the donor site without PRF.

\section{DISCUSSION}

Donor site discomfort and raw wound is the main drawback for the procedure of FGG. Protection of the donor site reduces the discomfort and pain. Formoush et al had described the various method to protect the donor site and decrease patient discomfort. ${ }^{1}$ they have described the use of interproximal ligature wire, stabilization of surgical dressing by 'mattress suture' and use of Hawley's appliance. ${ }^{1}$ However, these methods protect the donor site but do not promote the healing.

It was recently reported that PRF has positive effects on wound healing. PRF acts through several mechanisms, including the modulation of angiogenesis by growth factors that are found in the alpha granules of platelets. In addition, clot formation regulates neutrophil activity and causes faster 
epithelial cell migration to the wound area. These positive effects occur in response to growth factors and the stable fibrin scaffold. Therefore, fibrin has been referred to as a 'healing biomaterial' in the literature. ${ }^{2,5}$

Platelet-neutrophil interactions induce the neutrophils to release various antimicrobial molecules, such as lysozyme, myeloperoxidase and antimicrobial peptides called cathelicidins and $\alpha$-defensins. Neutrophils are also important in the regulation of wound healing and reepithelization. ${ }^{5}$

Soileau and Brannon reported that in conventional autogenous grafting procedures at least nine weeks are necessary for remodeling of the palatal wound to appear complete histologically. ${ }^{9}$ Alec Yen et al evaluated the effect of Platelet concentrate on palatal donor site healing after harvesting of sub-epithelial connective tissue graft and noticed that platelet concentrate could accelerate soft tissue healing and regeneration of palatal tissue thickness at 6-week intervals at clinical and histological levels. ${ }^{10}$

Various studies have reported that PRF enhances soft tissue healing in the donor site and reduces the patient discomfort.
Aravindaksha et al showed that the use of a PRF membrane as a palatal bandage is effective in accelerating soft tissue healing. ${ }^{6}$ Tamor et al also showed that PRF at donor site enhances healing and also reduces patient discomfort that occurs due to palatal plate. ${ }^{6}$ Kulkarni et al (2014) had conducted study in 18 patients and the result showed that sites where PRF was used showed complete wound closure by 14 days and these patients reported lesser post-operative morbidity than patients in whom PRF was not used. They concluded that the PRF as a dressing is an effective method of enhancing the healing of the palatal donor site and consequently reducing the post-operative morbidity. ${ }^{8}$ Reddy et al had concluded that the superior healing observed at the PRF membrane site supports its use in accelerating the soft tissue healing. PRF membrane as a bandage is an efficacious approach to protect the open wound in palate to reduce healing time and patient discomfort. ${ }^{11}$

The placement of PRF on the donor site after FGG enhance the healing of soft tissues and protect the raw wound and thus, reduces patient discomfort. It can be recommended for the wound coverage of donor site after free gingival graft.

\section{REFERENCES}

1. $\quad$ Farnoush A. Techniques for the protection and coverage of the donor sites in free soft tissue graft. J Periodontol. 1978;49(8):403-5.Shah R, Thomas R, Mehta DS. Recent modifications of free gingival graft: A case series. Contemp Clin Dent. 2015;6:425-7.

2. Femminella B, Iaconi MC, Di Tullio M, Romano L, Sinjari B, D'Arcangelo C, et al. Clinical comparison of platelet rich fibrin and a gelatin sponge in the management of palatal wounds following epithelialized free gingival graft harvest: a randomized clinical trial. J Periodontol. 2016 Feb;87(2):103-13.

3. Dohan DM, Choukroun J, Diss A, Dohan SL, Dohan AJ, Mouhyi J, Gogly B, et al. Platelet-rich fibrin (PRF):a second generation platelet concentrate. Part I: technological concepts and evolution. Oral Surg Oral Med Oral Path Oral Radiol Endod. 2006;101:E37-44.

4. Choukroun J, Diss A, Simonpieri A, Girard MO, Schoeffler C, Dohan SL, et al. Platelet-rich fibrin (PRF): a second-generation platelet concentrate. Part IV: clinical effects on tissue healing. Oral Surg Oral Med Oral Pathol Oral Radiol Endod. 2006;101:e56-e60.

5. Ustaoglu G, Ercan E Tunali M. The role of titanium-prepared platelet-rich fibrin in palatal mucosal wound healing and histoconduction. Acta Odontol Scand. 2016;74(7):558-64.

6. Aravindaksha SP, Batra P, Sood V, Kumar A, Gupta G. Use of platelet-rich fibrin membrane as a palatal bandage. Clinic Adv Periodontics. 2013;4:246-50.

7. Tomar N, Singh R, Jain G, Kaushik M, Dureja D. Enhancement of healing of donor hard palate site using platelet-rich fibrin. J Curr Res Sci Med. 2016;2:132-5.

8. Kulkarni MR, Thomas BS, Varghese JM, Bhat GB. Platelet-rich fibrin as an adjunct to palatal wound healing after harvesting a free gingival graft: A case series. J Indian Soc Periodontol. 2014;18:399-402.

9. Soileau KM, Brannon RB. A histologic evaluation of various stages of palatal healing following subepithelial connective tissue grafting procedures: A comparison of eight cases. J Periodontol. 2006;77:1267-73.

10. Yen CA, Griffin TJ, Cheung WS, Chen J. Effects of Platelet Concentrate on Palatal Wound Healing After Connective Tissue Graft Harvesting. J Periodontol. 2007;78:601-10.

11. Reddy S, MGS Prasad, S Singh, P Krishnanand, Bhowmik N, N Ashwini. Enhancing palatal wound healing by using platelet rich fibrin membrane as fibrin bandage. Int J Applied Dent Sci. 2015;1(4):02-04. 